\title{
A logic programming approach to medical errors in imaging
}

\author{
Susana Rodrigues ${ }^{a, *}$, Paulo Brandão ${ }^{b}$, Luís Nelas $^{c}$, José Neves $^{a}$, Victor Alves ${ }^{a}$ \\ a Department of Informatics, University of Minho, 4710 - 057 Braga, Portugal \\ b Clipóvoa-Hospital Privado, Espírito Santo Saúde, 4490-592 Póvoa de Varzim, Portugal \\ c CIT - Centro de Imagiologia da Trindade, 4000 - 285 Porto, Portugal
}

\section{A R T I C L E I N F O}

\section{Article history:}

Received 15 March 2010

Received in revised form

17 June 2011

Accepted 17 June 2011

\section{Keywords:}

Medical error

Classification system

Medical imaging

Knowledge representation

\begin{abstract}
A B S T R A C T
Background: In 2000, the Institute of Medicine reported disturbing numbers on the scope it covers and the impact of medical error in the process of health delivery. Nevertheless, a solution to this problem may lie on the adoption of adverse event reporting and learning systems that can help to identify hazards and risks. It is crucial to apply models to identify the adverse events root causes, enhance the sharing of knowledge and experience. The efficiency of the efforts to improve patient safety has been frustratingly slow. Some of this insufficiency of progress may be assigned to the lack of systems that take into account the characteristic of the information about the real world. In our daily lives, we formulate most of our decisions normally based on incomplete, uncertain and even forbidden or contradictory information. One's knowledge is less based on exact facts and more on hypothesis, perceptions or indications.

Purpose: From the data collected on our adverse event treatment and learning system on medical imaging, and through the use of Extended Logic Programming to knowledge representation and reasoning, and the exploitation of new methodologies for problem solving, namely those based on the perception of what is an agent and/or multi-agent systems, we intend to generate reports that identify the most relevant causes of error and define improvement strategies, concluding about the impact, place of occurrence, form or type of event recorded in the healthcare institutions.

Results and conclusions: The Eindhoven Classification Model was extended and adapted to the medical imaging field and used to classify adverse events root causes. Extended Logic Programming was used for knowledge representation with defective information, allowing for the modelling of the universe of discourse in terms of data and knowledge default. A systematization of the evolution of the body of knowledge about Quality of Information embedded in the Root Cause Analysis was accomplished. An adverse event reporting and learning system was developed based on the presented approach to medical errors in imaging. This system was deployed in two Portuguese healthcare institutions, with an appealing outcome. The system enabled to verify that the majority of occurrences were concentrated in a few events that could be avoided. The developed system allowed automatic knowledge extraction, enabling report generation with strategies for the improvement of quality-of-care.
\end{abstract}

○ 2011 Elsevier Ireland Ltd. All rights reserved.

\footnotetext{
* Corresponding author. Tel.: +351 932473 876; fax: +351 253604471.

E-mail address: simrr1@gmail.com (S. Rodrigues).
}

1386-5056/\$ - see front matter @ 2011 Elsevier Ireland Ltd. All rights reserved.

doi:10.1016/j.ijmedinf.2011.06.005 


\section{Introduction}

Currently, there is a growing awareness regarding the problem of medical error and how it affects both the service and the healthcare institution's quality or, in other words, the patient safety. According to a study undertaken by the European Commission in 2005 and published in the following year, about $78 \%$ of the inquired citizens classified medical errors as a major problem in their countries [1].

The Institute of Medicine's 2000 report, "To Err Is Human: Building a Safer Health System", focused attention sharply on medical error and patient safety. The conclusion that more people may die as a result of medical errors in hospitals than from injuries sustained in motor vehicle accidents is alarming. The report documented that medical errors cause between 44,000 and 98,000 deaths annually in the United States. In addition, medical errors result in annual costs of $\$ 17-\$ 29$ billion $[2,3]$.

It is widely recognized that we may learn more from our mistakes than from our successes. However, there is an apparent failure of healthcare systems to learn from mistakes. Too often healthcare providers do not advise others when a mishap does occur, nor do they share what they have learnt. As a consequence, the same mistakes occur repeatedly and patients continue to be harmed by preventable errors. One solution to this problem is reporting. At least, reporting can help to identify hazards and risks, and to provide information on the aspects that should be improved [4].

The main purpose of reporting systems is learning from experience. However, it is important to note that only the registration of errors is not sufficient to ensure patient safety. It is the response to errors that leads to change. The accumulation of potentially relevant data in databases contributes little to the improvement of healthcare. A technical specialized analysis of the data is required to identify trends and patterns [4-6].

An error can be defined as the failure of a planned action to be completed as intended or the use of a wrong plan to achieve an aim, and includes problems in practice, products, procedures, and systems [7].

It is important to note that classification systems work best when restricted to a specific medical field (e.g. medication errors, events of inadequate dialysis, transfusions) [4]. Although our approach has broad application in the medical field, we intended to focus the system on the adverse events happening in the medical imaging department. The medical imaging is a high-risk field for the occurrence of errors, especially due to the multiplicity of techniques, to the several stakeholders and to the complexity of the whole circuit that involves the conduct of studies. In modern Medicine, imaging studies play an important role in clinical practice. Most of the issues identified in studies on quality and safety in healthcare apply to Diagnostic Imaging. In the last two decades the greater sophistication and complexity of medical technology has led to an increase of errors. Communication, interpretation and perceptual errors, are some of the most common errors in medical diagnostic imaging that can lead to e.g., misdiagnosis, wrong-side examinations, wrong-name errors and delivering delay. The long learning curves of the new techniques, the inappropriate training, reliance on automated systems and confusing software features, are some of the relevant issues behind these problems [8,9].

In daily life, we make most of our decisions, if not all of them, based on incomplete, not precise, uncertain and even forbidden information. Knowledge is crucial to the problems of modern economy and society. In the scope of patient safety, medical error and adverse event reporting and learning systems there are several situations where information is insufficient or incomplete.

Unfortunately, most of information systems just ignore this characteristic of the information about the real world and build upon models where some idealisation expunges the inherent uncertainty [10]. The result is a system that never provides the expected answers, due to its inability to model the world. Instead, one should deal with the uncertainty in the model itself. Indeed, to implement useful information systems, namely knowledge based ones, it is necessary to represent and reason with defective information. Several approaches to the representation of imperfect knowledge may be found in literature. Many of them link logic with the theory of probabilities, combining Bayesian reasoning, Certainty Factors, Dempster-Shafer theory, Fuzzy Logic or non-standard logics [11-13].

The most effective way to prevent adverse events is to attack directly their causes [8]. It is common that one cause is also, somehow, a cause of various accidents. Preventing the adverse events' root causes improves significantly the patient safety [14]. Thus, our system focuses sharply on preventing the adverse events' root causes by applying a model that we've developed specifically for the medical imaging field. The model served as the formal foundation to our adverse event reporting and learning system, which is now deployed in two Portuguese healthcare institutions. The system makes possible to build on judgements about the impact, place of occurrence, type of form and type of event recorded in the healthcare institutions. It was possible to detect the adverse events that need immediate attention, identify its causes and generate recommendations to improvements.

In the next section, the classification system developed specifically for the medical imaging field and the clinical and theoretical concepts behind the system are presented. Based on the formal approach presented in this section, an adverse event reporting and learning system was developed. Section 3 presents the system, as well as the results obtained in the two Portuguese healthcare institutions where it was implemented. Finally, in the last section, conclusions are presented.

\section{Methods}

A medical version of the Eindhoven Classification Model (ECM) followed by the extensions and adaptations to the ECM for the medical imaging field and its causal tree that is used to classify the adverse events' root causes is presented. The theoretical foundation based on an extension to Logic Programming, in terms of a revision of its knowledge representation and reasoning system is described. By the introduction of explicit negation, leading to a process of on-the-fly quantification of the Quality of Information (QoI) of the predicates extensions 


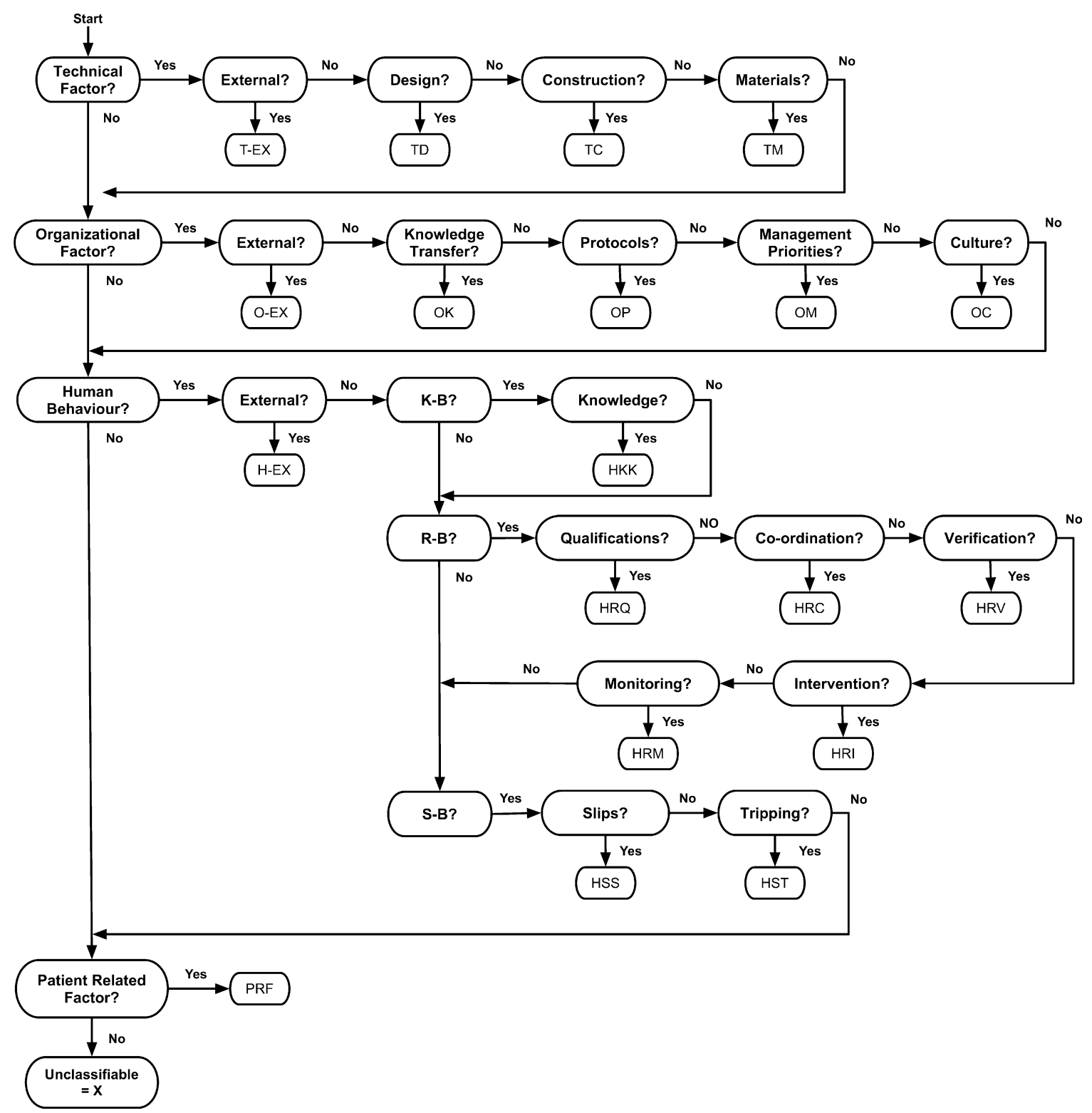

Fig. 1 - Eindhoven Classification Model - medical version classification flow chart.

Adapted from van Vuuren et al. (1997) [18].

that make a logic program and theory, the possibility to study the event's root causes and to generate alerts and recommendations on quality improvements enabled.

\subsection{The Eindhoven Classification Model}

A large number of different systems have been used to classify events regarding to patient safety [15].

In the past, the lack of a standard taxonomy lead to the adoption of different taxonomies that often hinder or prevent the sharing of data among systems. As a result, in 2005 the World Health Organization (WHO) started to focus its attention in the development of an internationally agreed taxonomy of events [4]. In January of 2009, the WHO released the final technical report "The Conceptual Framework for the International
Classification for Patient Safety - Version 1.1" [16]. This was our source for terms and concepts enabling future integration with other systems.

Many of the methods used to analyze patient safety were adapted from risk-management techniques in industries, especially in high-risk industries such as the chemical, nuclear power and aviation industry [14]. The Eindhoven Classification Model (ECM) was originally developed to manage human error in the chemical process industry and was then applied to various other industries, such as steel industry, energy production and in healthcare. The ECM medical version (Fig. 1) consists of 20 codes, divided into four categories (Table 1), frequently used in a medical environment to classify the underlying causes of the adverse events [17]. A full version of Table I with all the ECM categories and descriptions is available as supplementary 
Table 1 - Categories of the Eindhoven Classification Model - medical version.

\begin{tabular}{lc} 
Category & Code \\
\hline Technical & \\
External & T-EX \\
Design & TD \\
$\ldots$ & $\ldots$ \\
Organizational & \\
External & O-EX \\
Culture & OC \\
$\ldots$ & $\ldots$ \\
Human behaviour & \\
External & H-EX \\
Knowledge-based behaviour & \\
Knowledge-based errors & HKK \\
Rule-based behaviour & \\
Coordination & HRC \\
Intervention & HRI \\
$\ldots$ & $\ldots$ \\
Skill-based behaviour & $\ldots$ \\
Slips & \\
Tripping & HSS \\
Other & HST \\
Patient related factor & \\
Unclassifiable & PFR \\
\hline Adapted from Mers (2001) and van Vuuren et al. (1997) [14,18]. \\
\end{tabular}

material. The main feature of ECM is to base the prevention measures on the so-called system approach to the problem of human error. This approach assumes that humans are fallible and that errors are to be expected in every organization, so it's necessary to concentrate efforts on the conditions under which individuals work and try to build defences to avert errors or to mitigate their effects. Assigning codes to the causes of each adverse event are useful for tracking and trending [9,18-20].

Several studies present the application of the ECM in different medical fields (e.g. blood transfusion, cardiology, surgery, emergency) and in specific errors (e.g. thyroid gland fineneedle aspiration, tubing misconnections) [14,21-24].

The first step when using an ECM based classification system is to identify the root causes that result in a specific adverse event or noncompliance. These root causes are subsequently classified according to the ECM. To do so, a causal tree is built and techniques of Root Cause Analysis (RCA) are applied [14,25]. Once the root causes are identified, they can be used to provide a more realistic view of how the system really works, as well as to contribute to the creation of effective and lasting solutions $[14,17,18]$. Each root cause is classified into one of the ECM categories. The major advantage is that the classification, as can be seen in Fig. 1, focus firstly on system failures, rather than human ones. The category in which root cause is classified gives a clear view of the aspect that should be improved.

\subsection{Extended Eindhoven Classification Model}

The Extended Eindhoven Classification Model (EECM) was adapted from the ECM, presented in the previous section. To apply this model specifically to the medical imaging field, extensions were developed for each category of the original model. These extensions allow fitting each category into the medical imaging field and providing a broader view of the events' that may occur and the degree of complexity of this field. Some examples, for each pre-defined classification category were also included, in order to identify adverse events that meet the definition and the scope of each category. Thus, the classification process becomes easier and more efficient [25]. In Table 2 a subset of the EECM and some examples of events for the classification model's categories are presented. A full version of Table 2 is available as supplementary material.

For instance, in the original model, the adverse events classified as "Human behaviour - Knowledge-based errors" (HKK) occur due to "the inability of an individual to apply existing knowledge to a novel situation". In the specific field of medical imaging, we extend this definition by saying that the events classified under this category are due to "difficulties in execution, interpretation or reporting studies". Some of the adverse events falling into this category are "poorly executed reports, false positives, false negatives and reports poorly validated".

The causal trees taken on by the original ECM put up on hand the recognition of the event's root causes and its mental picture under a hierarchical structure. On the other hand, once one has to deal with incomplete and even contradictory information, we look to an Extension of Logic programming, namely Extended Logic Programming (ELP), to knowledge rep-

\section{Table 2 - ECM codes - medical imaging extended version (subset) [25].}

\begin{tabular}{lll} 
Code & \multicolumn{1}{c}{ Extension to Medical Imaging field } & \multicolumn{1}{c}{ Examples } \\
\hline T-EX & $\begin{array}{l}\text { Equipment's malfunction (e.g. CT, MRI, printers). } \\
\text { Failure of computer systems: digital dictation system, } \\
\text { heating/ventilation system. }\end{array}$ & $\begin{array}{l}\text { Studies not saved (necessary repeat the study). } \\
\text { Studies not performed. }\end{array}$ \\
OC & Lack of errors reporting systems. & Incapacity to react in adverse situations. \\
& Services not hierarchical. & \\
& Absence of procedure's rules. & \\
& Absence of periodic audits. & Report poorly executed. \\
& Lack of training in the area of security. & False positives and false negatives. \\
& Absermed consent not established. & Reports poorly validated. \\
DKK & &
\end{tabular}




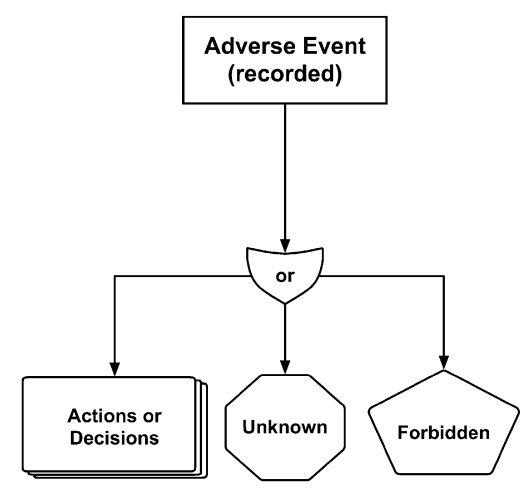

(A)
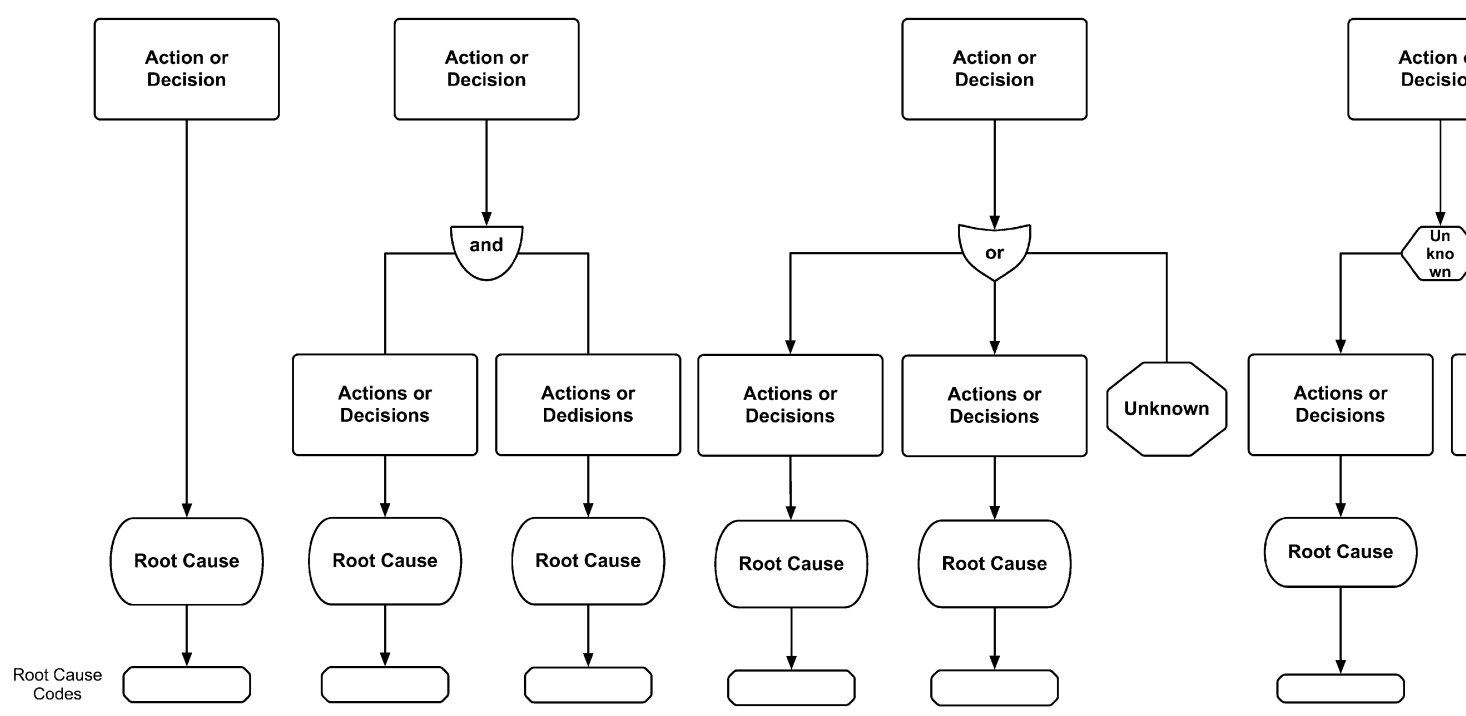

Fig. 2 - Structure of a causal tree extended with incomplete information operators: (A) general structure; (B) all possible cases.

resentation and reasoning, in order to get a truth value or measure of confidence in any qualification process susceptible to be handled by the system. Since, an event may only occur due to the combination of more than one cause and a different event may come about due to two or more causes, taken separately, in the original model "AND-gates" and "ORgates" are used, respectively, to embody these two possibilities in the causal tree. The usual situations can also include the case where only one cause leads to the occurrence of a certain event. In any of these cases the adverse events origins are known, i.e., there is a certainty about the events grounds. Beyond these situations, can also come about that the causes of an event, action or decision are unknown; may be known that certain views are the source of a given event, but may not be sure what are the event grounds; or it is not allowed to know the basis of a given event (e.g., due to internal policies of the healthcare institution).

Therefore, it is proposed the use of "unknown" and "forbidden" operators, to allow for the representation of unknown values of an infinite set of values, unknown values of a given set of values and values not allowed or forbidden (Fig. 2). The information contained in each causal tree is then represented in ELP by the extensions of a predicate set. Furthermore, it was adopted as a formalism to quantify the QoI present in each causal tree (see Section 2.3). The QoI allows for the identification of the causes that should be taken into account, in first place, and how they hamper all the classification process. The information obtained in this way to the Root Cause Analysis (RCA) enables automatic report generation with improvement recommendations.

Fig. 3 presents the application of the EECM to the adverse event "study not available for delivery on time". The selection of this event is related to its high frequency of occurrence verified by our reporting and learning system installed in two Portuguese healthcare institutions. In the source of this event there is a great diversity of reasons. It's possible that only one situation could be enough for the event to occur or, perhaps, it may be necessary a combination of several factors.

These extended causal trees are built in close collaboration with highly skilled healthcare professionals. All the actions, decisions and root causes are subject of an exhaustive study before getting the final structure of the causal trees. The causal trees include all possible causes and aim to be a generic representation of the problem. For a particular occurrence of the event, its causes will fall on a branch of the tree. 


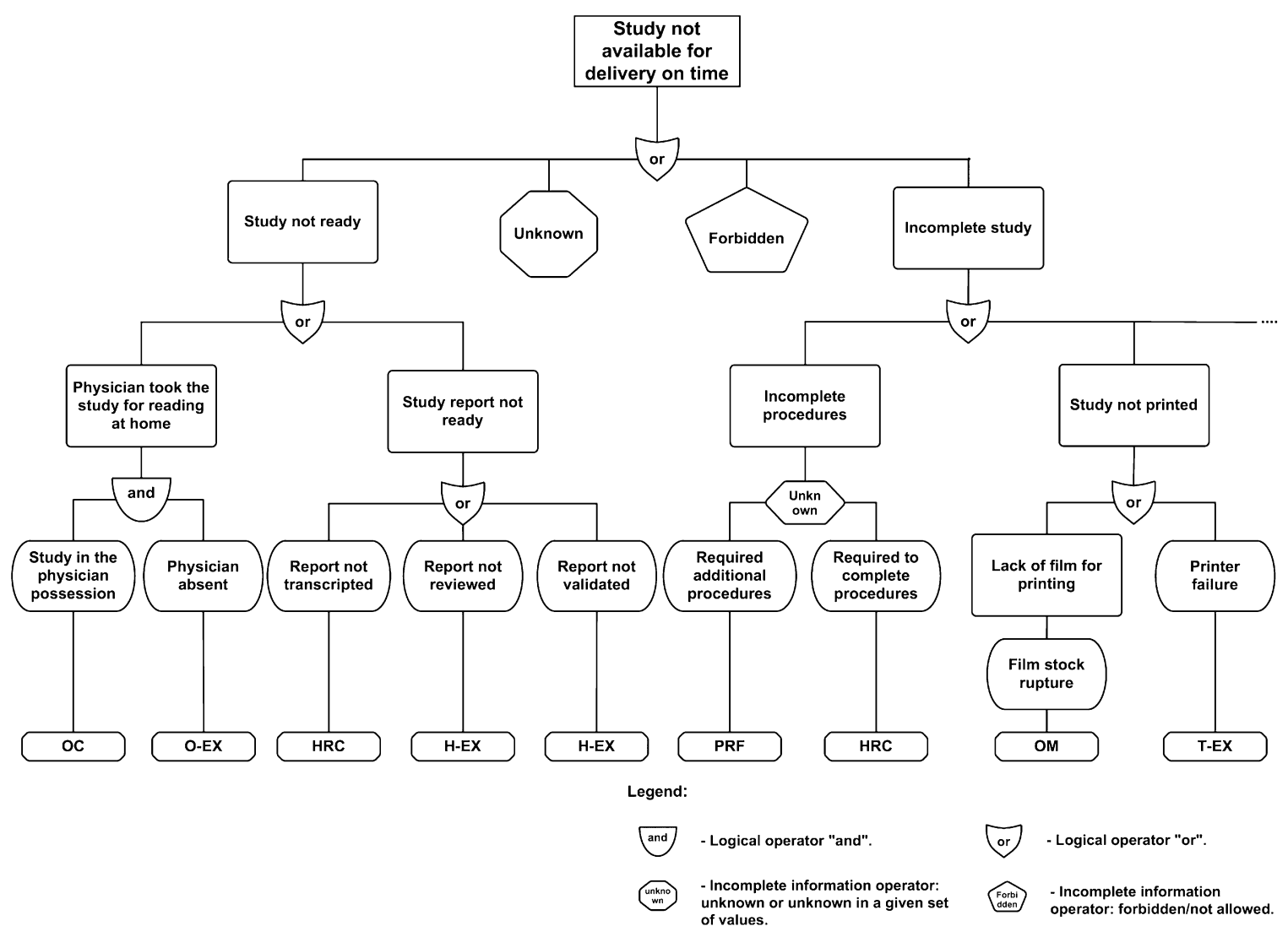

Fig. 3 - Extended causal tree for the adverse event "study not available to delivery on time".

\subsection{Knowledge representation and Quality of Information}

Intelligent systems require the ability to reason with incomplete and even contradictory information, even in the most unnatural situations.

In a classical logical theory or logic program, the proof of a theorem (here understood as a question submitted to the classification system) the outcome is a truth value, namely true or false [30].

An Extended Logic Program (ELP), on the other hand, is a finite collection of rules of the form [31]. ELP introduces another kind of negation, strong negation, represented by the classical negation sign $\neg$. In most situations, it is useful to represent $\neg A$ as a literal, if it is possible to prove $\neg A$. In ELP, the expressions $A$ and not $A$, being $A$ a literal, are extended literals, while $A$ or $\neg A$ are simple literals. Intuitively, not $p$ is true whenever there is no reason to believe $p$, whereas $\neg p$ requires a proof of the negated literal.

Every program is associated with a set of abducibles. Abducibles can be seen as hypotheses that provide possible solutions or explanations of given queries, being given here in the form of exceptions to the extensions of the predicates that make the program.

The issue is providing expressive power for representing explicitly negative information, as well as to directly describe the Closed World Assumption (CWA) for some predicates, also known as predicate circumscription [32,33]. Three types of answers to a given question are then possible, i.e. true, false and unknown. The representation of null values will be scoped by the ELP. We consider three types of null values: the first will allow for the representation of unknown values, not necessarily taken from a given set of values, the second will represent unknown values taken from a given set of possible values and the third will define values that are not allowed or forbidden.

Taking the example of the adverse event "study not available to delivery on time" it could represent all the possible situations according to the following examples:

- It is known that the study was not available for delivery on time because it was not ready - known value.

- The adverse event encompasses some conduct and judgement on its foundation on which it is not possible to be clear (affirmative) - unknown value.

- The healthcare professional that reported the adverse event, did not know if the study report was not ready, once the report was not transcript, reviewed or validated. It is not possible to be constructive, concerning the action or truth value to consider. However, it is false that the action or decision could be different. This situation suggests that the lack of knowledge may be associated to a set of possible known values - unknown value in a finite set of values.

- And finally, namely due to internal policies of the healthcare institution, it is not permitted to know the causes of a given event - forbidden or not allowed values. 


$$
\begin{aligned}
& \mathrm{V}_{\text {action_or_decision }}\left(1^{\text {st }} \text { case }\right)=1 \\
& \mathrm{~V}_{\text {action_or_decision }}\left(2^{\text {nd }} \text { case }\right)=0 \\
& \mathrm{~V}_{\text {action or decision }}\left(3^{\text {rd }} \text { case }\right)=0.33
\end{aligned}
$$

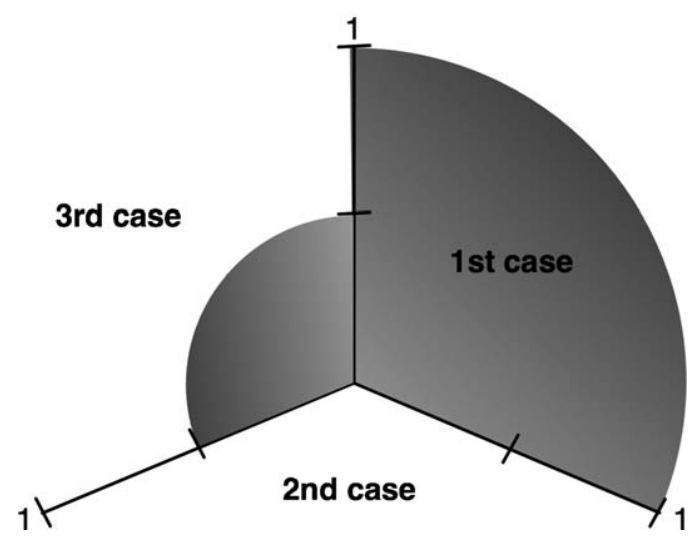

Fig. 4 - Quality of Information about the question "Which are the actions or decisions that led to the adverse event occurrence?".

Therefore, and using ELP, as the logic programming language, it is now possible to set a procedure given in terms of the extension of a predicate called demo:question,answer $\rightarrow[0,1]$. Given a question, it returns a truth-value based on a set of assumptions that will allow one to reason about the body of knowledge presented in a particular domain. Given a question, it returns a solution based on a set of assumptions, where question indicates a theorem to be proved and answer denotes a truth value (see Program 1; true (1), false (0), being $U$ in the range of the truth values]0,1[).

$$
\begin{aligned}
& \operatorname{demo}(Q, T) \leftarrow Q \\
& \operatorname{demo}(Q, F) \leftarrow \neg Q \\
& \operatorname{demo}(Q, U) \leftarrow \operatorname{not} Q \wedge \text { not } \neg Q
\end{aligned}
$$

Program 1. Extension of meta-predicate demo.

Indeed, in any decision making process, arguably, the decision is made without having all the information. What makes a decision maker to be confident about the reliability of the information?

Using the procedure at hand in the supplementary material, a picture as the one given in Fig. 4 to represent the reliability of the information concerned to a root cause analysis, is shaped. Here, the dashed n-slices of the circle denote de QoI that is associated with each of the predicate extensions that make the logic program.

As an example, we represent the QoI associated with the information about the RCA of the adverse event study not available to delivery on time, according to the three mentioned situations, depicted in Fig. 4. In order to find the relationships among the extensions of these predicates, we evaluate the relevance of the QoI, which is given in the form:

It is now possible to measure the QoI associated to the question put in context of the logic program, which was given in the form which are the actions or decisions that led to the adverse event occurrence?. The shaded $n$-slices (here $n$ is equal to 3 ) of the circle denote the QoI. A detailed description with practical examples of these issues is available as supplementary material.

\section{Results and discussion}

Based on the formal approach referred to above, on medical errors in imaging, an adverse event reporting and learning system (AEMI) was developed. Indeed, to the healthcare professionals, or the quality department and mostly for all the healthcare institution, this approach brings several advantages. After the adverse events being registered, similar to what happens in other reporting systems, the analysis process becomes easier, more expedite and reliable. Undoubtedly, with the recourse to ELP, leading to an on-the-fly measurement of the QoI of the logic terms used in the process of judgement (in terms of a theorem to be proved), the human intervention in the analyze process is only necessary to approve the recommendations, causes and events that need attention. It also caters for the credibility and the measurement of the efficacy of the implemented strategies and actions. The AEMI is a voluntary, confidential and non-punitive system, comprising three core modules, making it, not only a system for adverse event registration, but also a learning system, as mentioned above. Fig. 5 presents the overall view of the developed system.

Adverse Event Reporting Forms in Medical Imaging (AERFMI) module provides the Web interface for adverse event registration. The effort on this interface was focused in its usability. The event registration is made by professionals of the healthcare institution (e.g. physicians, administrators, radiology technicians) and by the public, through pre-defined forms adapted to each user profile reality. A screen shot of the Adverse Events and Near Misses Reporting Web Portal -AERFMI module can be seen in the supplementary material.

Adverse Events Manager Reports in Medical Imaging (AERMMI) module is also Web based and aims to enable the analysis of the adverse events recorded by AERFMI, based on the developed classification model. By applying the EECM the system allows to define improvement recommendations to reduce or eliminate the underlying organization failures. The system provides an individual report for each adverse event recorded which includes all its details and the extended causal tree obtained using the EECM. An example of the causal tree report is available in the supplementary material. These reports are automatically generated without human intervention. The user only has to access the needed report. Indeed, the event details are just a representation of what is stored in the system data base. The causal tree construction and the recommendations presented in the reports are only possible to be obtained thanks to the presented approach by applying the EECM and using ELP to knowledge representation and reasoning purposes.

These generated reports help the task of the quality department that use similar paper based reports (see supplementary material). Moreover, these generated reports focus attention on the most important feature of the event: its causes. The time and hand-labour needed to examine all the registers 


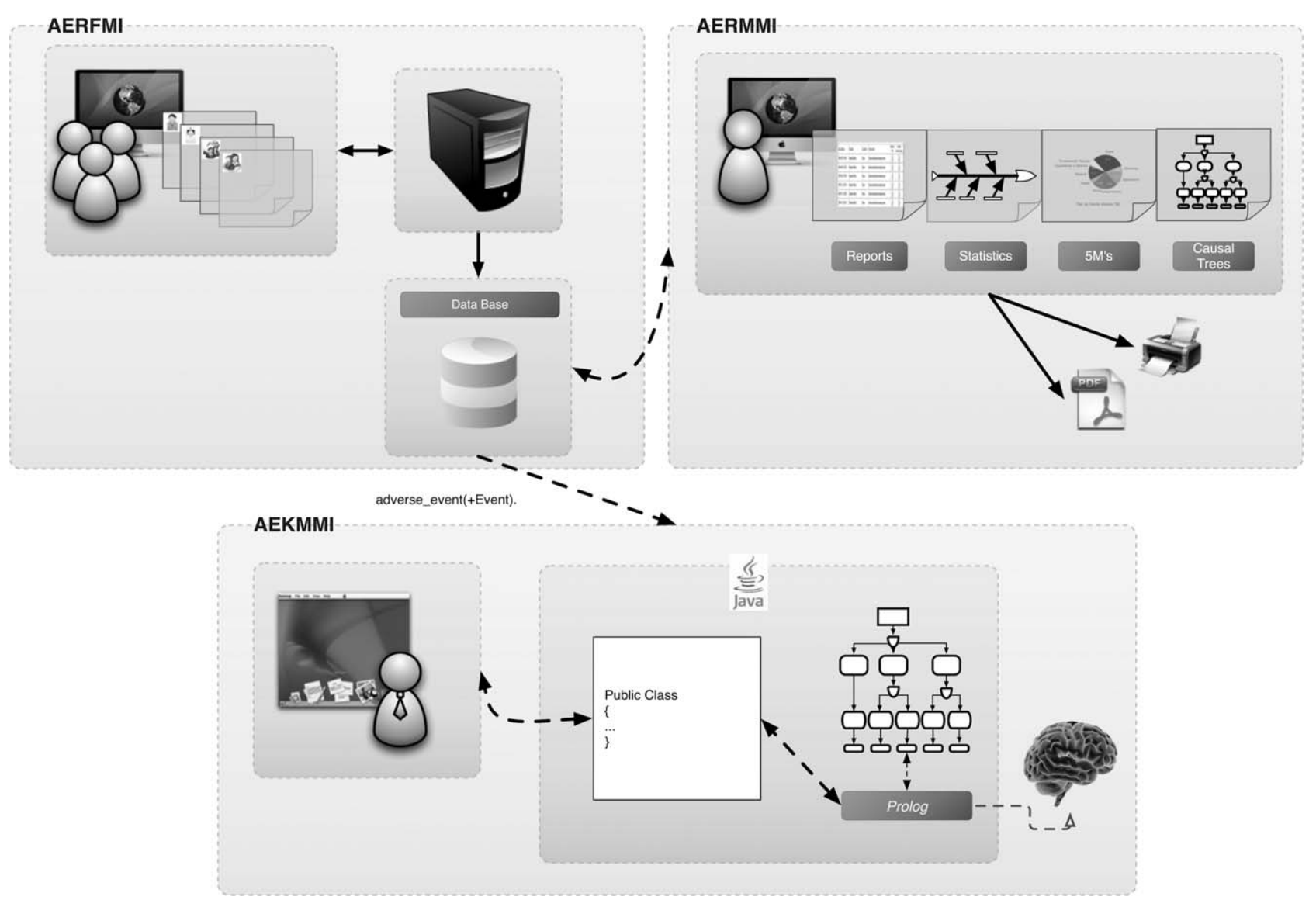

Fig. 5 - Overall view of the AEMI system.

at the same time, look for patterns and trends, is drastically reduced. All the information is stored in a common database that facilitates access for further processing, being object of a formal proof.

The AERMMI module also provides charts with statistical information about the impact, place of occurrence, type of form and type of event recorded. These charts are updated on the fly as more adverse events are registered and placed available online.

From recorded data in two Portuguese healthcare institutions it was possible to verify that the adverse event "study not available for delivery on time" had a much higher frequency than the other events registered through the system (event "E2" in Fig. 6), being a situation that deserved immediate attention. With the individual reports of each recorded event provide by the system it was possible to determine what actions and causes contribute the most to this adverse event occurrence. It is important to note that these actions and causes were identified by the system and presented to the users by the reports generated through the AERMMI module. The main cause was "study in the physician possession", because "physician took the study for reading at home". By applying the EECM it was highlighted the need to change the Organizational Culture (code OC from the EECM). This need was also automatically included in the system's reports, as happens to all the needs and recom- mendations identified by the system to all the adverse events registered.

In the specific case of the adverse event "study not available for delivery on time" measures were taken to assure that the studies do not leave the healthcare institution; the radiologist started to receive automatically a SMS warning, $48 \mathrm{~h}$ before the delivery deadline; $24 \mathrm{~h}$ before the delivery deadline ends the study will be read by another radiologist and a SMS was sent to inform the first radiologist.

It was possible to verify that the impact of the adverse events recorded in the two healthcare institutions is, mostly, mild (Fig. 7(A)). This impact is a measure of the degree of harm to the patient. Despite the impact of adverse events, such as "study not available for delivery on time", be typically mild, the financial costs that such event brings to the healthcare institutions, related not only with technical aspects but also with the quality image of the institution, is enough to be crucial to prevent its occurrence and take immediate actions. It was also possible to verify that most of the events occur with the Ultrasound modality (Fig. 7(B)).

In the two healthcare institutions, the users with the physicians' role were the ones that made the higher number of records (Fig. $7(C)$ ). Although physicians frequently show an aversion to this type of systems, this aversion was not notorious. Perhaps because it was made very clear that this system does not intend to be a measure of individual performance, but 


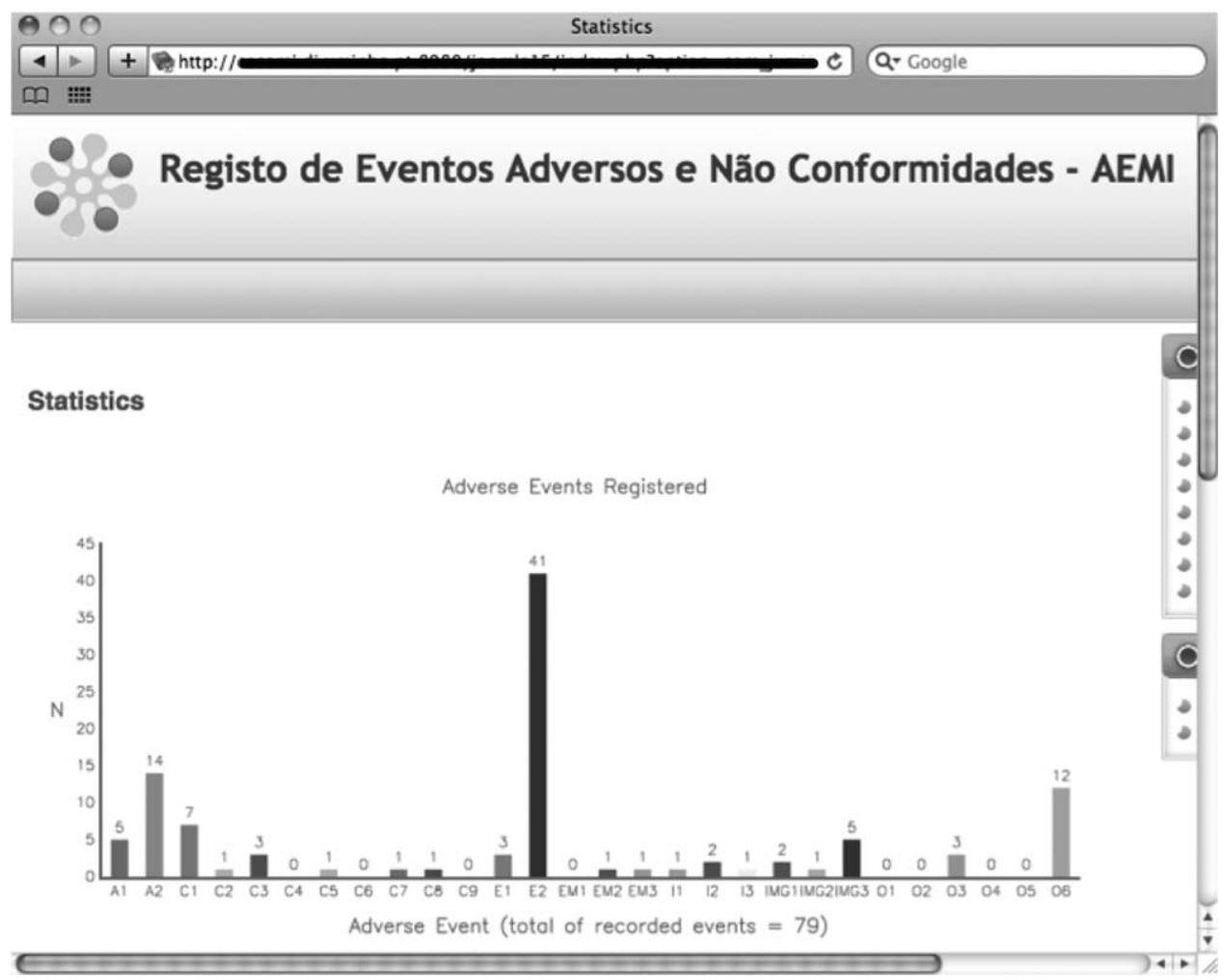

Fig. 6 - Screenshot of statistical analysis available in the Web interface of the AEMI: frequency of adverse events recorded.

rather a tool for improvement. On the other hand, the users with a radiology technician's role were the staff that less used the system. The public and the administrators adhered moderately. One major advantage of the developed system is the fact of a patient can register an adverse event. Often a patient has a completely different view of the adverse event, thus, his opinion is a valuable contribution to properly understand the event and its causes.

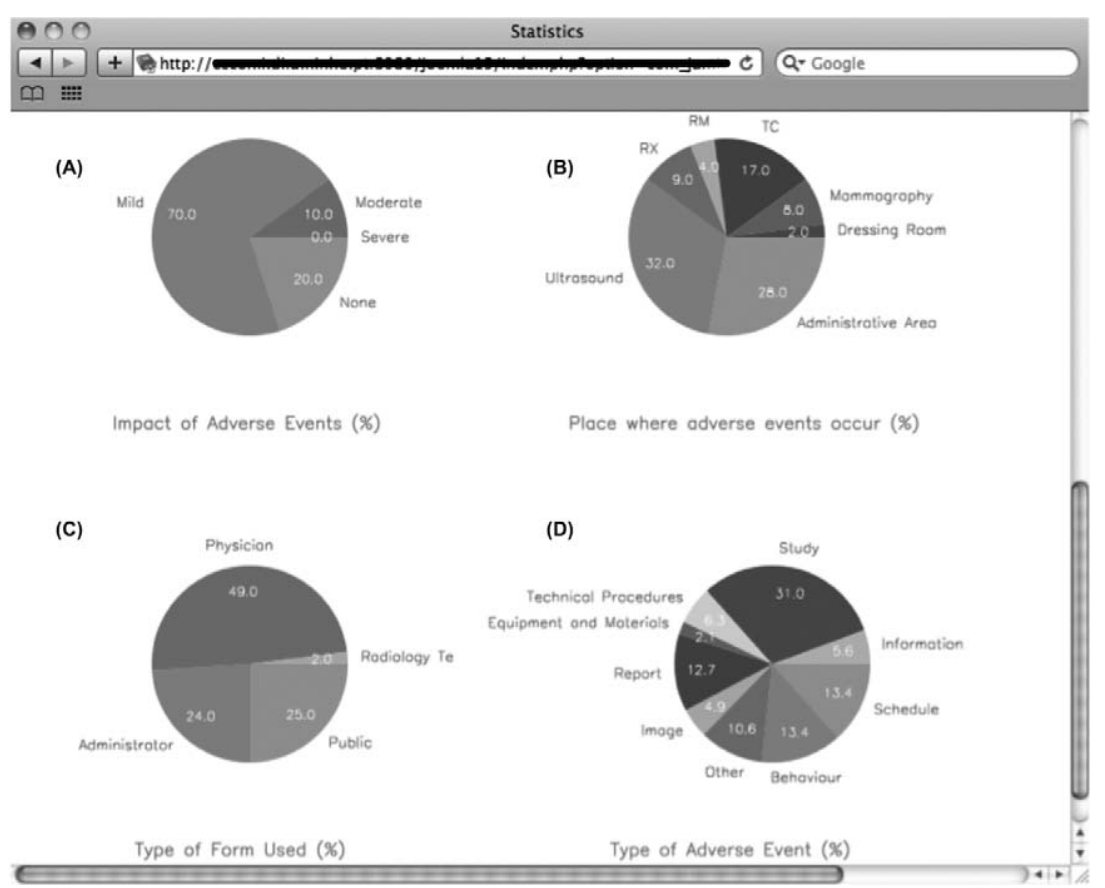

Fig. 7 - Screenshot of statistical analysis available in the Web interface of the AEMI: (A) percentage of events registered by impact; (B) percentage of events registered by place of occurrence; (C) percentage of events by the registration form used; (D)) percentage of events registered by type. 
The most frequent type of adverse event recorded was the study type (Fig. 7(D)), which implies that it is essential to review and redefine some of the procedures and methods used.

The Knowledge Manager Adverse Events in Medical Imaging (AEKMMI) module is a Java application. This module uses the data from the system database to create a Knowledge Base (KB), which although had been given in terms of ELP, is now rewritten to productions in the logic programming language Prolog, based on the EECM. From the KB other reports relevant to the improvement of the healthcare service can be generated, always with the assurance of the data reliability and credibility by taking into account the QoI. A screen shot of the AEKMMI interface is available as supplementary material.

\section{Conclusions}

The main contribution of this work is to be understood in terms of the evaluation of the QoI in the RCA and the possibility to address the issue of incomplete information, through the use of an extension to Logic Programming in the construction of causal trees. ELP was used for knowledge representation and reasoning with defective information, catering for the modelling of the universe of discourse in terms of incomplete, inconsistent, forbidden and default data and knowledge. A systematisation of the body of knowledge's evolution about QoI embedded in the RCA was made. A way to solve the representation problem of defective information was presented, adequate for evaluation the QoI in such process. It was also presented a computationally feasible formal tool to measure the value of QoI.

Although the causal classification of events is sometimes time-consuming and difficult, with the development of a generic causal tree for each possible event, the increase in time consuming is on the initial phase of the model application. The QoI allows for the ordering of causes, identifying the ones that should be taken into account in the first place. In the generic tree it is necessary to consider all possible causes, rather than most probable or usual ones. The information obtained is useful in identifying possible trends and areas requiring further investigation.

The conceptualized logic model offers the means for knowledge extraction, providing the identification of the most significant causes and suggestions of changes in the healthcare organization policies and procedures, subject to formal proof. Indeed, the creation of an inference system in support of the logical model enables the generation of reports with strategies for quality improvement on-the-fly, with a measure of the systemis confidence on the results, in terms of the QoI, i.e., an adverse event reporting and learning system was developed for medical errors in imaging. This system was deployed in two Portuguese healthcare institutions presenting useful results. The system enabled to verify that the majority of occurrences were concentrate in a few events that could be avoided. With our approach to RCA and classification it was possible to identify the causes, actions and decisions that lead to the adverse events and define the strategies to prevent them.

\section{Summary points}

Existing knowledge on medical errors and patient safety:

- There is an apparent failure of healthcare systems to learn from mistakes. One solution to this problem is reporting.

- The accumulation of potentially relevant data in databases contributes little to the improvement of healthcare.

- Classification systems work best when restricted to a specific medical field. The most effective way to prevent adverse events is to attack directly their causes.

- Most the systems ignore the characteristic of the information about the real world and build upon models where some idealization expunges the inherent uncertainty

What this study added:

- An adverse event reporting and learning system for medical imaging based on a specific classification model process focus on finding the underlining system failures is much more efficient in preventing the adverse events once the real root causes are identified and measures are put into action to enhance the system quality.

- The extension of ECM to the medical imaging field taking into account the systems failures and the adverse events root causes proved to be a useful tool to identify the system aspect of the healthcare institution that should be improved.

- The use of logic operators in the causal trees construction and the evaluation of the QoI in the RCA process allowed modelling the real world where incomplete and uncertain information prevails.

- A logic representation of the information contained in the causal tress highlights the knowledge extraction and enables the automatic generation of reports with strategies for quality improvement.

- With the implemented system it was possible to automatically conclude about the impact, place of occurrence, type of form and type of event recorded in the healthcare institutions, and detect the adverse events that need immediate attention, identify its causes and generate improvement recommendations.

\section{Author contributions}

Susana Rodrigues worked on the project analysis and development and in all computer implementations. She was also involved on the validation and the evaluation process and prepared the first version of the manuscript. Paulo Brandão was responsible for all medical aspects of the study. He collaborated in the validation and assessment of all proposed models and results, and later contributed to the revision of the paper. Luís Nelas was responsible for the data acqui- 
sition and causal trees development and also contributed to the paper revision. José Neves worked on the knowledge representation and reasoning processes of the model under construction. He was responsible for the final approval of the version to be submitted. Victor Alves had the coordinating task. He worked on the modelling and knowledge representation and contributed with important intellectual content to the paper.

\section{Conflict of interest}

None declared.

\section{Acknowledgements}

We are indebted to Clipóvoa - Hospital Privado and CIT Centro de Imagiologia da Trindade, for providing their adverse events dataset and for their help in terms of experts and technicians.

\section{Appendix A. Supplementary data}

Supplementary data associated with this article can be found, in the online version, at doi:10.1016/j.ijmedinf.2011.06.005.

\section{REFERENCES}

[1] European Commision. Medical Errors, Special Eurobarometer. 2006 [cited 2009 Jul 8]. Available from: ec.europa.eu/health/ph_information/documents/eb_64. en.pdf.

[2] L. Kohn, J. Corrigan, M. Donaldson, To Err is Human: Building a Safer Health System, National Academy Press, Washington, DC, 1999.

[3] L. Leape, D. Berwick, C. Clancy, J. Conway, P. Gluck, J. Guest, et al., Transforming healthcare: a safety imperative, Qual. Saf. Health Care 18 (December (6)) (2009) 424-428.

[4] World Health Organization Alliance for Patient Safety, WHO Draft Guidelines for Adverse event reporting and learning systems, World Health Organization, Geneva, 2005.

[5] J.C.L. Kohn, M. Donaldson, To Err is Human: Building a Safer Health System, National Academy Press, 2000.

[6] World Health Organization Alliance for Patient Safety, Project to develop the international patient safety event taxonomy: updated review of the literature 2003-2005, World Health Organization, Geneva, 2005.

[7] United States, Quality Interagency Coordination Task Force, Doing what counts for patient safety: federal actions to reduce medical errors and their impact, report of the Quality Interagency Coordination Task Force (QuIC) to the President. Quality Interagency Coordination Task Force, Washington, DC, 2000.

[8] P. Brandão, A. Reis, Error in medical imaging - performance and computed detection systems, Acta Med. Port. 19 (2006) 235-238 (Portuguese)

[9] J. Reason, Human error: models and management, BMJ 320 (March (7237)) (2000) 768-770.
[10] S. Parsons, Current approaches to handling imperfect information in data and knowledge bases, IEEE Trans. Knowledge Data Eng. 8 (3) (1996) 353-372.

[11] B.G. Buchanan, E.H. Shortliffe, Rule Based Expert Systems: The Mycin Experiments of the Stanford Heuristic Programming Project (The Addison-Wesley series in artificial intelligence), Addison-Wesley Longman Publishing Co., Inc, Boston, MA, USA, 1984.

[12] G. Shafer, in: S.C. Shapiro (Ed.), The Dempster-Shafer theory Encyclopedia of Artificial Intelligence, second ed., Wiley, 1992.

[13] L.A. Zadeh, Logic Fuzzy, in: R.A. Wilson, F.C. Keil (Eds.), The MIT Encyclopedia of the Cognitive Sciences, MIT Press, 2001.

[14] T.M. Mers. Medical Event Reporting System for Transfusion Medicine Reference Manual Version 3.0. New York, 2001 [cited 2010 Jan 10]. Available from: http://mers-tm.org.

[15] A. Chang, P.M. Schyve, R.J. Croteau, D.S. O'Leary, J.M. Loeb, The JCAHO patient safety event taxonomy: a standardized terminology and classification schema for near misses and adverse events, Int. J. Qual. Health Care 17 (April (2)) (2005) 95-105.

[16] World Health Organization Alliance for Patient Safety, The Conceptual Framework for the International Classification for Patient Safety - Version 1.1. Final Technical Report, World Health Organization, Geneva, 2009.

[17] T.W. van der Schaaf, PRISMA-Medical: a brief description. Eindhoven University of Technology, Faculty of Technology Management, Patient Safety Systems, 2005.

[18] W. van Vuuren, C.E. Shea, T.W. van ser Schaaf, The Development of an Incident Analysis Tool for Medical Field Eindhoven, Eindhoven University of Technology, 1997.

[19] J.T. Reason, Human Error, Cambridge University Press, New York, 1990.

[20] J. Rasmussen, Outlines of a Hybrid Model of the Process Operator, Plenum Press, London, 1976.

[21] P.A. Nast, M. Avidan, C.B. Harris, M.J. Krauss, E. Jacobsohn, A Petlin, et al., Reporting and classification of patient safety events in a cardiothoracic intensive care unit and cardiothoracic postoperative care unit, J. Thorac. Cardiovasc. Surg. 130 (October (4)) (2005) 1137.

[22] E.A. Henneman, F.S. Blank, S. Gattasso, K. Williamson, P.L. Henneman, Testing a classification model for emergency department errors, J. Adv. Nurs. 55 (July (1)) (2006) 90-99.

[23] S.S. Raab, C.M. Vrbin, D.M. Grzybicki, D. Sudilovsky, R. Balassanian, R.J. Zarbo, et al., Errors in thyroid gland fine-needle aspiration, Am. J. Clin. Pathol. 125 (June (6)) (2006) 873-882.

[24] D. Simmons, K. Graves, Tubing misconnections - a systems failure with human factors: lessons for nursing practice, Urol Nurs. 28 (December (6)) (2008) 460-464.

[25] S. Rodrigues, Adverse Events and Near-Misses in Health Care. Department of Informatics, University of Minho, Portuguese, 2009.

[30] U. Hustadt. Do we need the closed-world assumption in knowledge representation? In: Baader, Buchheit, Nutt (Eds.), KI'94 Workshop, Saarbrüken, Germany, 1994.

[31] M. Gelfond, V. Lifschitz, Logic programs with classical negation, in: D. Warren, P. Szeredi, Logic Programming, Proceedings of the 7th International Conference, 1990, pp. 579-597.

[32] J. Neves, A logic interpreter to handle time and negation in logic data bases, in: Proceedings of the ACM'84, The Fifth Generation Challenge, 1984.

[33] K.R. Apt, R. Bol, Logic programming and negation: a survey, J. Logic Programming 19 (1994) 9-71. 